\title{
Assessment of Prescribing Pattern for Hypertension and Comparison with JNC-8 Guidelines-Proposed Intervention by Clinical Pharmacist
}

\author{
Sivasakthi Raju*, Sam Solomon, Nithiyan, Karthik, Anns Clara Joseph, Venkatanarayanan \\ Department of Pharmacy Practice, RVS College of Pharmaceutical Sciences, Coimbatore-641 402, INDIA.
}

\begin{abstract}
Background: Hypertension is a common disorder that if not effectively treated results in greatly increased probability of coronary thrombosis, strokes, and renal failures. It is estimated that the prevalence of hypertension in India is about 25\% among urban adults and $10 \%$ in the rural areas. The lifetime risk of developing hypertension is estimated to be $90 \% .^{1}$ To assess prescribing pattern in hypertensive patients and assessing the rationality in regards to JNC-8 Guidelines in a tertiary care hospital. Methodology: It was a prospective, observational study conducted for 6 months with of 75 hypertensive prescriptions with diabetes mellitus and/ or chronic kidney disease who get admitted in the hospital were screened for the study. Result: In a total of 75 prescriptions, 14 (18.7\%) prescriptions were found in the age group of less than 50 years, in which $10(13.3 \%)$ were males and $4(5.4 \%)$ were females and $36(48 \%)$ prescriptions were comes under the age group between $51-65$ years, in which $26(34.7 \%)$ were males and $10(13.3 \%)$ were females. Also 25 (33.3\%) prescriptions were comes under the age group of above 65 years, in which $20(26.7 \%)$ were males and $5(6.7 \%)$ were females. Among mono therapy $22(47.8 \%)$ prescriptions had diuretics as antihypertensive drug followed by CCB 11 $(24 \%)$, ARBs $7(15.1 \%)$, $\beta$ blockers $3(6.5 \%)$, ACEls $1(2.2 \%)$, $\alpha$ blockers $1(2.2 \%)$ and $\alpha+\beta$ blockers $1(2.2 \%)$. When compared with JNC 8 Guidelines it was observed that $28 \%$ of prescriptions were rational and $56 \%$ of prescriptions were irrational. Regarding the perception of physicians towards JNC 8 guidelines it was found that majority were not following JNC 8 guidelines.

Key words: Hypertensive's, Combination therapy, Co-morbidity, JNC 8
\end{abstract}

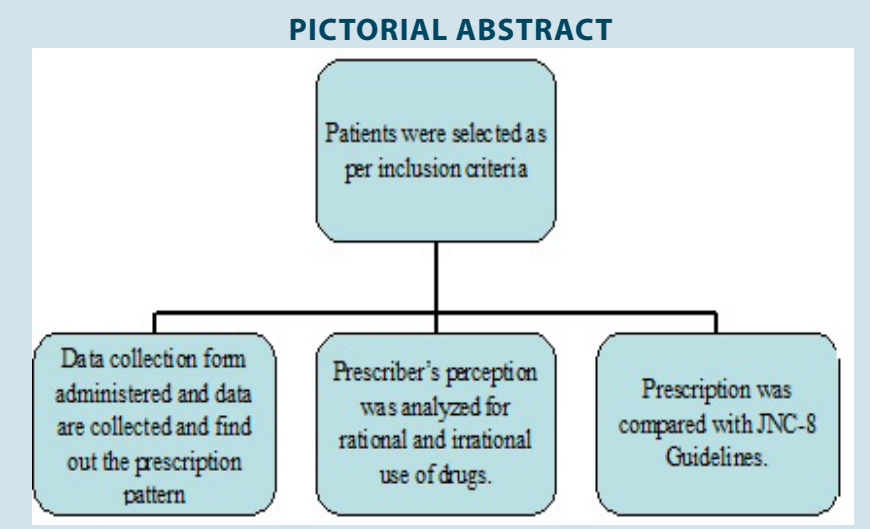

Guidelines.

Correspondence :

Sivasakthi Raju

Department of Pharmacy Practice, RVS College of Pharmaceutical Sciences, Coimbatore-641 402, INDIA.

Phone no: +91-9965141122

E-mail: sivasakthimpharm@gmail.com

DOI: $10.5530 /$ jyp.2016.2.14

\section{INTRODUCTION}

Hypertension is a common disorder that if not effectively treated results in greatly increased probability of coronary thrombosis, strokes, and renal failures. It is estimated that the prevalence of hypertension in India is about $25 \%$ among urban adults and $10 \%$ in the rural areas. The lifetime risk of developing hypertension is estimated to be $90 \%{ }^{1}$

Hypertension is a common healthcare problem. The prevalence among adult population has been estimated to range from $4.75 \%$ to $25.6 \%$ and constitutes $1.8 \%$ to $3.8 \%$ of total consultations in primary health center. Because of various types of adverse reactions, benefit, contraindications, and cost of the selection of appropriate drug is very important one. ${ }^{2}$ High Blood pressure remains a Major risk factor for premature Death and disability. $62 \%$ of Stroke and $49 \%$ of Myocardial Infarctions has been attributed with Increased risk of elevated Blood pressure. ${ }^{3}$

As per JNC-8 Report, persons Older than 50 years, Systolic blood pressure of More than $140 \mathrm{mmHg}$ is a much more important Cardio vascular disease and it recommends Life style modifications to prevent Cardio vascular disease. Thiazide diuretics are used for Uncomplicated Hypertension. Followed by ACEIs, Angiotensin Blockers receptors, Calcium channel blockers are used. The study reports that, the Control of Hypertension was improved by motivation when patient have positive experience with and Trust in the Clinicians. ${ }^{4}$ Polypharmacy was observed as one of the reason for variations in disease state. ${ }^{19}$

\section{CAUSES}

As per JNC-8, the causes of Hypertension are Improper Blood pressure Measurement, Volume Over loaded, Pseudo tolerance, Excess sodium intake, Non-adherence, Inadequate doses of drugs, Inappropriate combinations, Amphetamines, Sympathomimetic, Oral contraceptives and Tobacco. Obesity and excess alcohol intake are associated causes of Hypertension. ${ }^{4}$ The DASH diet eating plan has been proven to lower blood pressure in studies sponsored by the National Institute of Health. The DASH plan incorporated more fruits and vegetables, low fat or non-fat dairy, beans, and nuts than the others studied. The diet reduced systolic blood pressure by $6 \mathrm{mmHg}$ and diastolic blood pressure by $3 \mathrm{mmHg}$ in patients with normal blood pressure. ${ }^{1}$ The main purpose of this study is to assess prescribing pattern in hypertensive patients and assessing the rationality in regards to JNC-7 Guidelines and perception of physicians about JNC 8 guidelines in a tertiary care hospital.

\section{METHODOLOGY}

It was a prospective, observational study conducted for 6 months. The study was conducted at KG Hospital and Post Graduate Medical Institute, Coimbatore, a 350-bedded tertiary care hospital. A total of 75 hypertensive prescriptions with diabetes mellitus and/or chronic kidney disease who get admitted in the hospital were screened for the study. 


\section{GENDER DISTRIBUTION}

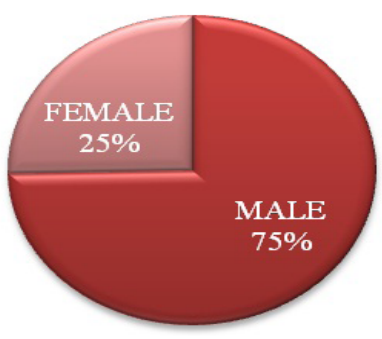

Figure 1: Gender Distribution

Patient data collection form was administered successfully and data were analyzed by using SPSS software.

\section{RESULTS}

This prospective, observational study on assessment of prescription pattern for hypertension and comparison with JNC 8 Guideline was conducted in KG Hospital and Post Graduate Medical Institute, Coimbatore, in which 75 prescriptions were screened for the study. The results are as follows:

In this study, Figure 1 show that out of 75 prescriptions 56 (75\%) prescriptions were for males and 19 (25\%) prescriptions were for females.

In a total of 75 prescriptions, $14(18.7 \%)$ prescriptions were found in the age group of less than 50 years, in which 10 (13.3\%) were males and $4(5.4 \%)$ were females and $36(48 \%)$ prescriptions were comes under the age group between 51-65 years, in which 26 (34.7\%) were males and 10 (13.3\%) were females. Also 25 (33.3\%) prescriptions were comes under the age group of above 65 years, in which 20 (26.7\%) were males and $5(6.7 \%)$ were females. out of 75 prescriptions, $50(67 \%)$ patients had DM as co-morbidity, 10 (13 \%) had CKD and 15 (20\%) had both CKD and DM as co-morbidities.

In our study, we observed that $8(10.7 \%)$ patients who are less than 50 years had DM as co-morbidity, 5 (6.7\%) had CKD and $1(1.3 \%)$ had both CKD and DM. Patients in the age group between 51-65 years were observed that $24(32.0 \%)$ had DM, 2 (2.7\%) had CKD and 10 (13.3\%) had both CKD and DM. In patients above 65 years the pattern of co-morbidities are as follows, DM 18 (24\%), CKD 3 (4\%) and CKD + DM $4(5.3 \%)$. The study results that $46(61 \%)$ patients were prescribed with mono therapy. The remaining 29 (39\%) were prescribed with combination therapy. Among mono therapy $22(47.8 \%)$ prescriptions had diuretics as antihypertensive drug followed by CCB 11 (24\%), ARBs 7 (15.1\%), $\beta$ blockers 3 (6.5\%), ACEIs 1 (2.2\%), $\alpha$ blockers 1 (2.2\%) and $\alpha+\beta$ blockers 1 (2.2\%). In two drug combinations 5 (17.2\%) prescriptions had $\mathrm{CCB}+\alpha$ blockers combination followed by 4 (13.8\%) prescription had CCB+Loop diuretics, 4 (13.8\%) had $\beta$ blockers+Loop diuretics. When considering three drug combination it was found that a combination of loop diuretics+CCB+ $\alpha$ blockers was prescribed for $2(6.9 \%)$ patients, followed by $1(3.5 \%)$ had loop diuretic $+\mathrm{CCB}+(\alpha+\beta$ blockers $)$ and loop diuretic $+\mathrm{ARB}+\alpha$ blockers each. Two prescriptions were found with four drug combination.
When compared with JNC 8 guidelines for hypertension, it was found that $42(56 \%)$ of prescriptions falls into the group of irrational, $12(16 \%)$ of prescriptions were semi rational and $21(28 \%)$ of prescriptions were rational. When comparing relationship between co-morbidities and rationality $21(28 \%)$ prescriptions were following JNC8 guidelines (rational) out of which $17(22.6 \%)$ patients having DM as co-morbidity and $42(56 \%)$ prescriptions were irrational out of which $31(41.3 \%)$ patients having DM as co-morbidity. When coming to semi-rational prescriptions, $7(9 \%)$ having both co-morbidities. When considering the relation between therapy and rationality, it was observed that $21(28 \%)$ prescriptions were according to JNC8 guidelines out of which 19(25\%) prescriptions having mono therapy and $2(3 \%)$ having combination therapy. When considering irrational prescriptions (42(56\%)) 27(36\%) prescriptions were mono therapy and $15(20 \%)$ were combination therapy. There is no prescription having mono therapy in semi-rational and $12(16 \%)$ having combination therapy.

To analyze the perception of physicians towards the applicability of JNC 8 Guidelines over JNC 7 Guidelines in a clinical setting, a questionnaire was prepared containing 5 questions and distributed to physicians prescribing antihypertensive drugs. From the data obtained, it was known that $7(35 \%)$ of physicians were comfortable in using JNC 8 whereas $13(65 \%)$ were not comfortable in adapting JNC 8 guidelines while prescribing antihypertensive medicine.

\section{CONCLUSION}

This prospective, observational study on assessment of prescribing pattern for hypertension confirmed that mono therapy was prescribed than the combination therapy. Specify with the guidelines, Loop diuretics were the most frequently prescribed drug class followed by CCB, ARBs, beta blockers, ACEIs, Alpha blockers and $\alpha+\beta$ blockers. When considering combination therapy, two drug combination was prescribed more than three drug and four drug combinations. In two drug combination, $\mathrm{CCB}+\alpha$ blockers was mostly prescribed. Diabetes mellitus is the most commonly occurring co-morbidity in hypertensive patients. When compared with JNC 8 Guidelines it was observed that $28 \%$ of prescriptions were rational and $56 \%$ of prescriptions were irrational. Regarding the perception of physicians towards JNC 8 guidelines it was found that majority were not following JNC 8 guidelines. The assessment of perception of physicians towards stated guidelines shows that $65 \%$ of the physicians were comfortable in adapting to JNC 8 guidelines while prescribing antihypertensive medicines. So, a more effective update of the knowledge is necessary. It also suggests a need of compliance by clinicians with JNC 8 guidelines. This confirms the need for clinical pharmacist in each department and aware the physicians to improve the adherence to new guidelines in clinical setting.

\section{ACKNOWLEDGEMENT}

The Authors thanks to Dr. G. Bakthavathchalam, Chairman, KG Hospital and post graduate research Institute, Coimbatore, for Ethical Committee Clearance ( dated : 10 $0^{\text {th }}$ January 2014) and Guidance.

\section{CONFLICT OF INTEREST}

The author declare no conflict of interest. 


\section{ABBREVIATIONS USED}

JNC-8: Joint National Committee Report-8, CCB: Calcium channel blockers, ACEI: Angiotensin converting enzyme Inhibitors, CKD: Chronic kidney disease, DM: Diabetes mellitus.

\section{ABOUT AUTHORS}

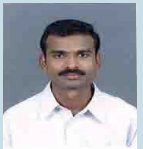

R. Sivasakthi: Is a doctoral student at the The TN Dr. MGR Medical University, Chennai, where he graduated in Bachelor of Pharmacy and Master of Pharmacy. His doctoral research focused on the Assessment of Prescription pattern and Comparison with JNC-8 Guidelines as well as Measuring the Drug related Outcomes. Has experience in the area of Pharmacovigilance and Therapeutic drug monitoring.

W. D. Sam Solomon: Obtained his PhD Degree at Javdapur University, Kolkata. he is positioned as Professor and Head at the Medicinal Chemistry \& Pharmaceutical Analysis Department of RVS College of Pharmaceutical Sciences, Coimbatore, (India). Dr. W.D.Sam Solomon is working on various aspects of Bio-analytical method development od drugs by LC-MS/MS and HPLC as well as Preparation of bio analytical validation study report.

\section{REFERENCES}

1. Vishal DJ, Dahake AP. Adverse effects Associated with the Use of Antihypertensive Drugs: An Overview. International Journal of Pharmaceutical Technology and Research. 2010;2(1):10-3.

2. Bader A. Almustafa; Use of anti-hypertensive medications: an Educational need in Saudi Primary Health Care; Middle East Journal of Family Medicine. 2006;4(5):5-7.

3. MC Alister, Feldman, Katherine, Rollin B. The Impact of the Canadian Hypertension Education Programme in its First Decade. European Heart Journal. 2009;30: 1434-39

4. Aran V. The Seventh Report of the Joint National Committee on Prevention, Detection, Evaluation, and Treatment of High Blood Pressure. Journal of American Medical Association. 2003;289(19):2560-87.

5. William EM, Eve MC. Blood Pressure Screening of School Children in a Multiracial School District: The Healthy Kids Project. American Journal of Hypertension. 2009;22(4):351-6

6. Arain MI. A Case Study on Pharma co vigilance and Prescribing trend of Antihypertensive therapy in Government Hospital of Hyderabad. Pakistan IJBPAS. Dec-2013;2(12):2449-59.

7. Srinivasan R, Ramya G. Adverse Drug Reaction-Causality Assessment. IJRPC. 2011;1(3):2231-34.

8. Dabhade SB, Atre K. Review on Pharma co vigilance study of Telmisartan in Hypertension Patients. AJPCR. 2013;6(3):974-5.

9. James M. The Effect of Clinical Pharmacy Services on Patients with Essential Hypertension. Circulation. 1973;48(5):1104-1111.

10. Sanjay DCA. Adverse drug reactions and their risk factors among Indian ambulatory elderly patients. Indian Journal of Medical Research. 2012;136(3):404-10.
11. Sharma HS. A Pharma co vigilance study in the department of medicine of university teaching hospital. Pharmacy Practice. 2007;5(1):46-9.

12. MR L, Wald NJ, Morris JK, Jordan RE. Value of Low dose Combination treatment with blood pressure lowering drugs. British Medical Journal. 2003;326 (28):1427.

13. Adigun AQ. Shifting trends in the Pharmacologic treatment of hypertension in Nigerian tertiary hospital: a real-world evaluation of the efficacy, safety, rationality and Pharma co economics of old and newer antihypertensive drugs. Journal of Human Hypertension. 2003;17(4):277-85.

14. Liamis G, Milionis $H$, Elisaf M. Blood pressure drug therapy and electrolyte disturbances. International Journal of Clinical Practice. 2008;01;1572-9.

15. Nilima AK, Shirsagar SK. Adverse Drug Reaction Monitoring in Pediatric Practice Journal of Indian Pediatrics. 1996;33(12);993-6.

16. Jagannadha R. Cardiac Biomarkers: The Troponins and CK-MB. Ibnosia Journal of Medicine and Biomedical Sciences. 2010;2(5):190-7.

17. Lica M. Troponin concentrations $72 \mathrm{~h}$ after myocardial infarction as a serological estimate of infarct size. Journal of Heart. 2002;87:520-4.

18. Mari M, Hideki M, Yukio Y. Nutritional biomarker Analyses for Hypertension related Risk Assessment and Control. World Journal of Cardiovascular Disease. 2014;4:268-76.

19. Shelat PR. A Study of Drug Utilization Pattern according to Daily Define Dose in Intensive Care Unit (ICU)s at Tertiary Care Teaching Hospital, India. Journal of Young Pharmacists. 2015;7(4):349-58

20. Viral NS. Drug utilization pattern and pharmacoeconomic analysis in geriatric medical in-patients of a tertiary care hospital of India. Journal of Pharmacology and Pharmacotherapeutics. 2014;5(2):166. 